\title{
Nitric Oxide Stimulates Elastin Expression in Chick Aortic Smooth Muscle Cells
}

\author{
Hideki Sugitani, ${ }^{a}$ Hiroshi Wachi, ${ }^{*}, a$ Shingo Tajima, ${ }^{b}$ and Yoshiyuki Seyama ${ }^{a}$ \\ Department of Clinical Chemistry, Hoshi College of Pharmacy, ${ }^{a}$ 2-4-41 Ebara, Shinagawa-ku, Tokyo 142-8501, Japan \\ and Department of Dermatology, National Defense Medical College, ${ }^{b}$ 3-2 Namiki, Tokorozawa, Saitama 359-8513, Japan. \\ Received December 4, 2000; accepted February 16, 2001
}

\begin{abstract}
Nitric oxide (NO), an endothelium-dependent relaxing factor, regulates relaxation, proliferation, and migration of smooth muscle cells (SMCs) and most likely attenuates developing vascular disease such as atherosclerosis. We investigated whether or not NO is associated with regulation of aortic elasticity. $S$-Nitrosoglutathione (GSNO), a NO donor, stimulated tropoelastin synthesis in cultured SMCs during both the quiescent and proliferating phases. The stimulation of tropoelastin synthesis was dose-dependent within $1-100 \mathrm{~nm}$. Maximum stimulation was detected by treatment with $100 \mathrm{~nm}$ GSNO for $24 \mathrm{~h}$. 8-Bromoguanosine $3^{\prime}, 5^{\prime}$-cyclic monophosphate (8Br-cGMP), an exogenous cyclic GMP analog, also upregulated tropoelastin synthesis. Tropoelastin and lysyl oxidase mRNA expression, as assessed by Northern blot analysis, was also stimulated by GSNO. Administration of KT5823, a cyclic GMP-dependent protein kinase inhibitor, inhibited the GSNO-induced tropoelastin synthesis. These results indicate that the stimulatory effects of GSNO are due to cyclic GMP dependent protein kinase (PKG) activation by NO. In conclusion, NO seems to enhance aortic elasticity via tropoelastin and lysyl oxidase upregulation.
\end{abstract}

Key words elastin; nitric oxide; lysyl oxidase; cGMP; smooth muscle cell

The protein elastin is responsible for the characteristic elastic properties of many tissues including skin, lung, and large blood vessels. Elastin is secreted from the cells as a soluble monomer, tropoelastin. It is believed that tropoelastin is associated with several other proteins such as microfibrils, and then it cross-links with other tropoelastin molecules when activated by lysyl oxidase to form elastic fibers. ${ }^{1)}$ Tropoelastin expression is modulated by a number of factors, including transforming growth factor-beta (TGF-beta), ${ }^{2)}$ insulin like growth factor, ${ }^{3)}$ interleukin-1, ${ }^{4)}$ epidermal growth factor (EGF), ${ }^{5}$ and minoxidil. ${ }^{6}$ However, the mechanism regulated by NO remains unknown.

In atherosclerotic plaques, mature elastic fibers are degenerated, and there are decreased amounts of cross-linked amino acids, such as desmosine or isodesmosine, produced by lysyl oxidase. ${ }^{7)}$ As a result, the elastic properties of the aorta were decreased due to the increased disorder of the system. Recently, mice that lack elastin underwent subendothelial cell proliferation and reorganization of SMCs, both of which are similar to processes seen in cases of atherosclerosis. ${ }^{8)}$ The data indicated that elastin exerts a regulatory function during arterial development; in particular, elastin controls cell growth in SMCs. Thus, it is important to investigate the mechanisms controlling elastin expression, including chemical factor, hormones, and cytokines in SMCs.

Nitric oxide, an endothelium-dependent relaxing factor, is continuously produced by NO synthase in the endothelium. ${ }^{9)}$ In addition, NO regulates platelet adhesion and aggregation, ${ }^{10,11)}$ leukocyte recruitment and activation, ${ }^{12)}$ and cytokine-induced endothelial cell activation, ${ }^{13)}$ as well as vascular smooth muscle cell apoptosis, ${ }^{14)}$ proliferation, ${ }^{15)}$ and migration. ${ }^{16)}$ Endothelial dysfunction causes decreased NO release, resulting in a shared process involving the pathogenesis of vascular diseases, including atherosclerosis, and neointima formation after angioplasty. ${ }^{17-19)}$ It is possible that NO is associated with atherosclerosis in elastic fiber formation as well as in cell proliferation and migration.
In the present study, we investigated whether or not GSNO, an NO donor, ${ }^{20)}$ upregulates elastin expression, as assessed by Northern blot analysis and metabolic labeling. We suggest that the upregulation by GSNO of tropoelastin expression is due to PKG. In this report, we clarify the NOcontrol mechanism governing the expression of elastin.

\section{MATERIALS AND METHODS}

Materials GSNO and 8-Br-cGMP were purchased from Sigma. KT5823 was obtained from Calbiochem. $\left[3,4-{ }^{3} \mathrm{H}\right]-$ Valine $(1.5 \mathrm{TBq} / \mathrm{mmol})$ and $\left[\alpha_{-}{ }^{32} \mathrm{P}\right] \mathrm{dCTP}(110 \mathrm{TBq} / \mathrm{mmol})$ were supplied by Amersham. Fetal bovine serum (FBS), valine-free Dulbecco's modified Eagle's medium (DMEM), and Trypsin-EDTA were purchased from Gibco.

Cell Culture and the Effect of GSNO on Cell Proliferation Chick aortic smooth muscle cells were isolated from 20-d-old chick embryonic aortae by serial enzyme digestion with bacterial collagenase (Wako) and pancreatic elastase (Sigma), as previously described. ${ }^{21)}$ Cells were seeded at a density of $2.1 \times 10^{5} \mathrm{cells} / \mathrm{cm}^{2}$ in 35 or $100-\mathrm{mm}$-diameter culture dishes (Falcon Plastics); cells were grown to confluent density in DMEM supplemented with $10 \%$ FBS.

Confluent cells were incubated with serum-free DMEM for $48 \mathrm{~h}$ to induce the $\mathrm{G}_{0}$ phase, and then were treated with 0 , 1,10 , and $100 \mathrm{~nm}$ GSNO for $24 \mathrm{~h}$ in the absence or presence of bovine serum. Treated cells were harvested by trypsin $(0.25 \%)$ treatment. The cell number was counted by a Burker-Turk hemocytometer.

Metabolic Labeling and SDS-PAGE Confluent cells were cultured with FBS-free DMEM for $48 \mathrm{~h}$ to induce the $\mathrm{G}_{0}$ phase, and then were treated with various concentrations $(1-100 \mathrm{~nm})$ of GSNO for $24 \mathrm{~h}$ in $10 \%$ FBS-DMEM or FBSfree DMEM. The cells were labeled with $25 \mu \mathrm{Ci} / \mathrm{ml}$ of [3, 4${ }^{3} \mathrm{H}$ ] valine for the final $6 \mathrm{~h}$ of treatment in valine-free DMEM. The protein in the culture medium was precipitated with ammonium sulfate $(176 \mathrm{mg} / \mathrm{ml})$ in the presence of pro- 
tease inhibitors, $1 \mathrm{~mm}$ EDTA, NEM, and PMSF, and then was centrifuged at $10000 \mathrm{~g}$ for $20 \mathrm{~min}$ at $4{ }^{\circ} \mathrm{C}$. The proteins from the cultured medium were separated on $2-15 \%$ SDS-PAGE under the condition of reduction with $1 \mathrm{~mm}$ DTT. Samples were then subjected to fluorography. Fluorograms were scanned with a densitometer. Tropoelastin synthesis was evaluated by the ratio of tropoelastin to total proteins.

RNA Isolation and Northern Hybridization Cultures were washed twice in PBS, and cells were lysed in $4 \mathrm{~m}$ guanidine isothiocyanate containing $25 \mathrm{~mm}$ sodium citrate and 0.1 $\mathrm{mm}$ beta-mercaptoethanol. RNA was isolated by extraction in phenol/chloroform. For the Northern hybridization analysis, $10 \mu \mathrm{g}$ of total RNA was denatured in glyoxal and DMSO at $50{ }^{\circ} \mathrm{C}$ for $1 \mathrm{~h}$ and samples were separated by electrophoresis on $1 \%$ agarose gels. RNA was passively transferred to nylonreinforced nitrocellulose membranes (Schleicher \& Schuell). The membranes were hybridized to ${ }^{32} \mathrm{P}$-labeled probes for chick tropoelastin, lysyl oxidase, or beta-actin, which were prepared with rediprime ${ }^{\mathrm{TM}} \mathrm{II}$, a random prime labelling system (Amersham). Chicken elastin (pTE2) ${ }^{22}$ and beta-actin $(\mathrm{pA} 1)^{23)}$ were used as cDNA for ${ }^{32} \mathrm{P}$-labeled probes. $300 \mathrm{bp}$ of chick lysyl oxidase cDNA $^{24}$ were amplified by Reverse Transcriptase-Polymerase Chain Reaction with a sense primer (5'-ACGGACGATAACCCCTACTACAACT-3') and an anti-sense primer ( $5^{\prime}$-CGCACTATGTTGTTGGAGTAATCAG-3'). The filters were washed first in a medium with a stringency of $2 \times \mathrm{SSC} / 0.1 \% \mathrm{SDS}$, and then in $0.1 \times \mathrm{SSC} / 0.1 \%$ SDS kept at $65^{\circ} \mathrm{C}$ for $60 \mathrm{~min}$. The filters exposed to X-ray film (Kodak) at $-80^{\circ} \mathrm{C}$ with an intensifying screen (Kodak). The autoradiograms were scanned with a densitometer. The mRNA expression of tropoelastin and lysyl oxidase was normalized by that of beta-actin.

\section{RESULTS}

GSNO Inhibited Cell Proliferation To confirm the effects of GSNO on SMC proliferation in FBS-DMEM or FBS-free DMEM, quiescent cells were treated with GSNO for $24 \mathrm{~h}$ in the absence or presence of bovine serum. Administration of GSNO $(100 \mathrm{nM})$ in growth medium inhibited serum-stimulated cell proliferation by $50 \%$, whereas GSNO in FBS-free DMEM had no effect on the number of cells observed. (Fig. 1). Under these two different conditions, cytotoxicity was not detected (data not shown).

GSNO and 8-Br-cGMP Enhanced Tropoelastin Protein Synthesis The changes in tropoelastin protein synthesis in SMCs brought about by drug treatment were detected by SDS-PAGE following a metabolic labeling assay with $\left[{ }^{3} \mathrm{H}\right]$ Valine. Immunoblotting analysis using monoclonal antibody for tropoelastin revealed that the bands, which indicated with an arrow, are related to tropoelastin. ${ }^{25)}$ Moreover, we previously reported that bands of tropoelastin were confirmed by incorporations of radiolabeled cysteine and valine, but not incorporations of radiolabeled mannose, glucose, and methionine. ${ }^{26}$ Since complete amino acid sequence deduced from chicken cDNA demonstrated that tropoelastin has no sugar moiety nor methionine residue and has two cysteine residues only near the carboxyl-terminal end..$^{22,27,28)}$ In the absence of fetal bovine serum, tropoelastin protein synthesis was stimulated by GSNO in concentrations ranging from 1 to $100 \mathrm{~nm}$ (Figs. 2A, B). As was the case in the presence of

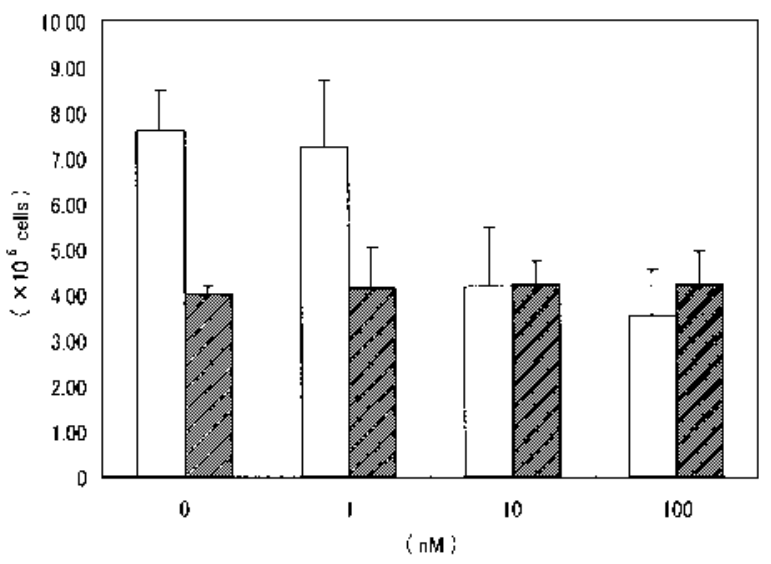

Fig. 1. Effect of GSNO on Cell Proliferation

Cell number was counted Burker-Turk hemocytometer. SMCs were incubated with $0,1,10$, and $100 \mathrm{~nm}$ GSNO for $24 \mathrm{~h}$ in the absence (striped bar) or presence (clear bar) of $10 \%$ bovine serum. The values indicate the means \pm range from quadruplicate experiments.
$A$
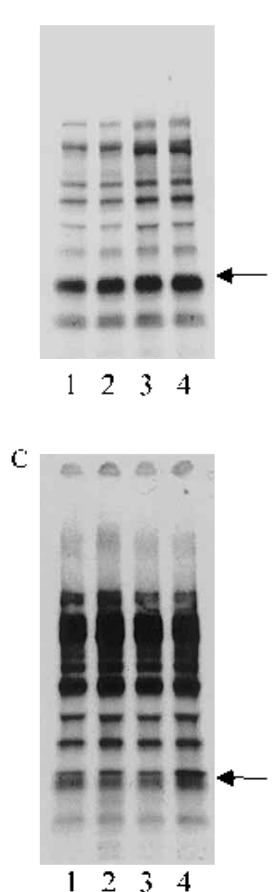

L

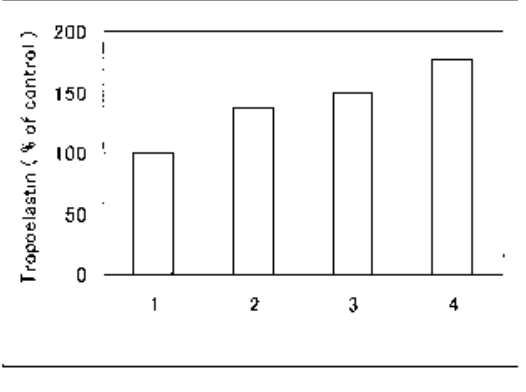

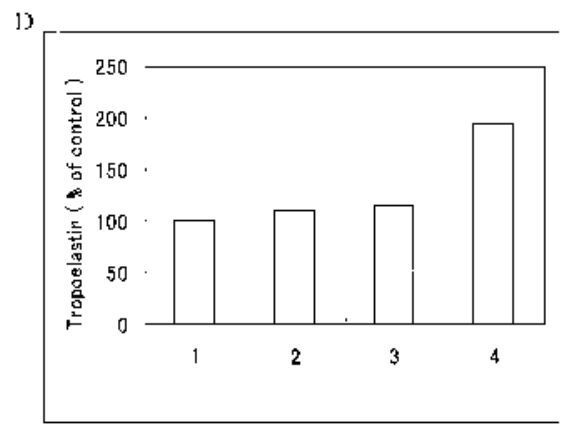

Fig. 2. Dose-Dependent Stimulation of GSNO on Tropoelastin Synthesis

Cells were treated for $24 \mathrm{~h}$ with 0 (lane 1), 1 (lane 2), 10 (lane 3), and $100 \mathrm{~nm}$ (lane 4) of GSNO in the absence (A, B) or presence (C, D) of FBS, and then labeled with $\left[{ }^{3} \mathrm{H}\right]$ valine for the last $6 \mathrm{~h}$ of treatment. The protein from the medium analyzed by SDS-PAGE, and visualized by autoradiography $(\mathrm{A}, \mathrm{C})$. The arrow indicates the position of tropoelastin. The autoradiograms were quantitated with a scanning densitometer (B, D). Values are mean from duplicate experiments.

serum, tropoelastin protein concentrations in SMC also increased due to treatment with GSNO (Figs. 2C, D). NO elevates cGMP levels by activating guanylate cyclase in affected cells. In order to examine whether or not cGMP was able to regulate elastin synthesis, cells were exposed to 8-Br-cGMP. Treatment with 8-Br-cGMP did enhance tropoelastin synthesis (Fig. 3).

GSNO Stimulated Tropoelastin and Lysyl Oxidase mRNA Expression Tropoelastin and lysyl oxidase mRNA expression, as assessed by Northern blot analysis, were stim- 
ulated by GSNO in concentrations ranging from 1 to $100 \mathrm{~nm}$ in the presence of FBS. Tropoelastin and lysyl oxidase mRNA concentrations increased about 1.4 -fold and 4.5 -fold, respectively, after 24-h exposure to $100 \mathrm{~nm}$ GSNO (Fig. 4).

Effect of GSNO on Tropoelastin Synthesis was Due to cGMP-Dependent Protein Kinase Activation In order to examine whether or not GSNO increased tropoelastin protein synthesis through the intermediary of PKG activation, a specific PKG inhibitor, KT5823, was used. A combination of KT5823 with GSNO attenuated the increase in tropoelastin, while treatment of GSNO (100 nM) stimulated tropoelastin synthesis, although administration of KT5823 (250 nM) alone showed increased tropoelastin synthesis (Fig. 5).

\section{DISCUSSION}

GSNO appears capable of stimulating elastin synthesis and mRNA expression in both experimental conditions, included in the present study, namely, in the absence and presence of FBS. However, GSNO inhibited cell proliferation of cells cultured in the presence of FBS and it had no effect on the number of cells cultured in the absence of bovine serum. Several reports have demonstrated that modulation of elastin synthesis by $\mathrm{EGF}^{5)}$ high potassium salt ${ }^{25)}$ or minoxidil ${ }^{6}$ is
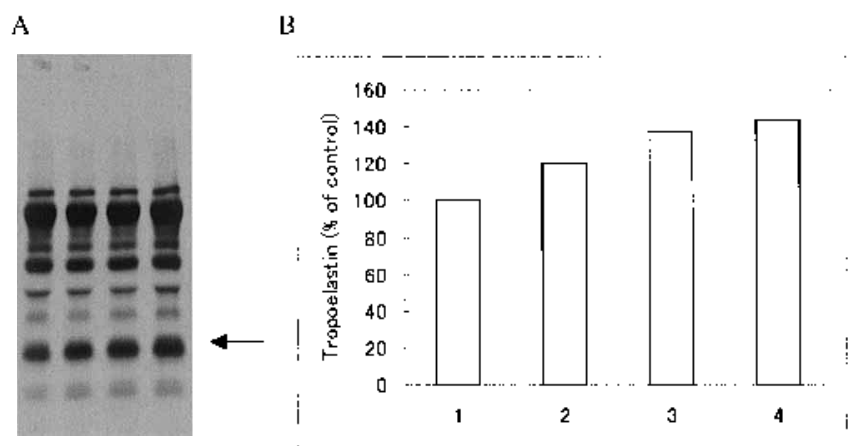

] $23 \quad 3 \quad 4$

Fig. 3. Dose-Dependent Stimulation of 8-Br-cGMP on Tropoelastin Synthesis

Cells were treated for $24 \mathrm{~h}$ with 0 (lane 1), 1 (lane 2), 10 (lane 3), and $100 \mu \mathrm{M}$ (lane 4) of 8 -Br-cGMP in the absence of FBS, and then labeled with $\left[{ }^{3} \mathrm{H}\right]$ valine for the last $6 \mathrm{~h}$ of treatment times. The protein from the medium analyzed by SDS-PAGE, and visualized by autoradiography (A). The arrow indicates the position of tropoelastin. The Autoradiograms were quantitated with a scanning densitometer (B). Values are mean from duplicate experiments. inversely associated with cell proliferation. However, the upregulation of tropoelastin synthesis due to GSNO differs among the existing reports. It thus appears as if NO regulates tropoelastin synthesis by mechanisms other than by the upregulation of tropoelastin.

In most studies of the antiproliferative effect of NO on smooth muscle cells, the process was mediated via a cGMPdependent pathway. ${ }^{15,29)}$ In a previous report, cGMP analog was shown to enhance tropoelastin synthesis in cultured fetal bovine ligamentum nuchae. ${ }^{30)}$ We confirmed that 8-Br-cGMP also has the capacity to upregulate tropoelastin synthesis in chick aortic smooth muscle cells. Moreover, KT5823 blocked GSNO-induced upregulation of tropoelastin, thus suggesting that GSNO activates PKG to enhance this synthesis. We have demonstrated that PKG activation is an important role on such upregulations due to GSNO. In general, the upregulating effects of $\mathrm{NO}$ on tropoelastin synthesis appears mediated by a cGMP-dependent mechanism which includes PKG.

In this study, we discuss GSNO stimulation of mRNA expression of lysyl oxidase as well as that of tropoelastin. Our

h

B
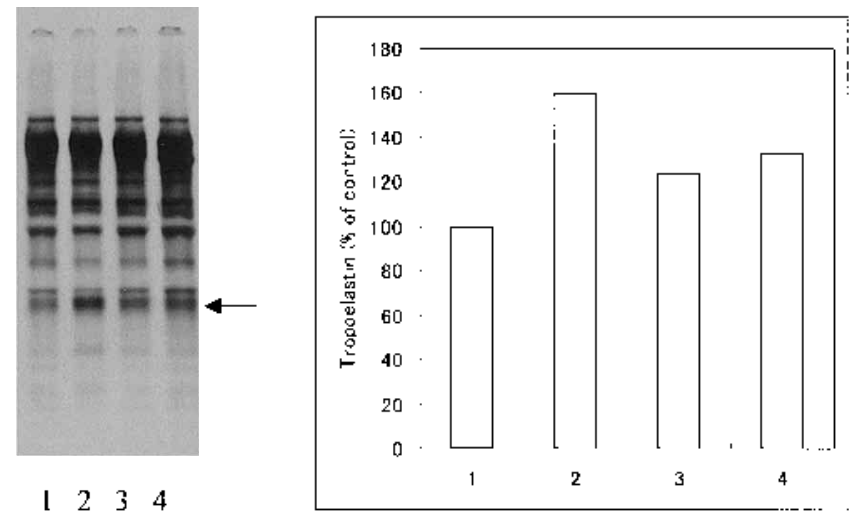

Fig. 5. Effect of KT5823 on GSNO-Induced Stimulation of Tropoelastin Synthesis

SMCs were treated with vehicle (lane 1), $100 \mathrm{nM}$ of GSNO (lane 2), $250 \mathrm{~nm}$ of KT5823 (lane 3), or combination of $100 \mathrm{~nm}$ of GSNO and $250 \mathrm{~nm}$ of KT5823 (lane 4) for $24 \mathrm{~h}$ in the presence of FBS, and labeled with $\left[{ }^{3} \mathrm{H}\right]$ valine for the last $6 \mathrm{~h}$ of treatment times. The protein from the culture medium analyzed by SDS-PAGE, and visualized by autoradiography (A). The arrow indicates the band of tropoelastin. The autoradiograms were quantitated with a scanning densitometer (B). Values are mean from duplicate experiments.
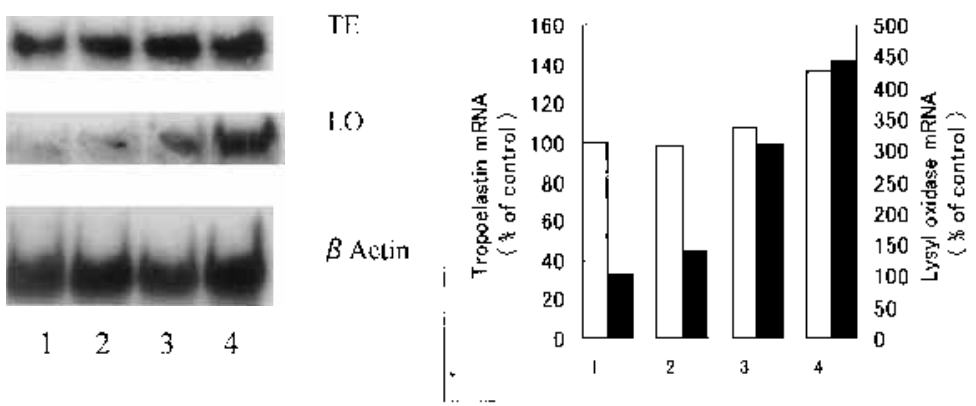

Fig. 4. Effect of GSNO on Tropoelastin (TE), Lysyl Oxidase (LO), and Beta-actin mRNA Levels in SMCs

RNA was extracted from cells treated for $24 \mathrm{~h}$ with 0 (lane 1), 1 (lane 2), 10 (lane 3), and $100 \mathrm{~nm}$ (lane 4) of GSNO in the presence of FBS. Ten micrograms of total RNA were separated on $1 \%$ agarose gel electrophoresis, and transferred onto membranes. Northern hybridization was performed with specific ${ }^{32} \mathrm{P}$-labeled cDNA probes, and visualized by autoradiography (A). The autoradiograms were quantitated with a scanning densitometer (B). Values are mean from duplicate experiments. 
results suggest that NO release increases the expression of both tropoelastin and lysyl oxidase. Tropoelastin crosslinks via lysyl oxidase, thus forming elastic fiber. As a probable result, NO may easily accelerate elastic fiber formation in the aorta. Because elastin biosynthesis plays a crucial role in maintaining tissue elasticity, the genes regulated by NO are important for persevering aortic function. A previous report demonstrated that cAMP stimulates the expression of lysyl oxidase in adult SMCs and inhibits the proliferation of SMCs. ${ }^{31)}$ These observations were confirmed by experiments including the addition of TGF-beta. ${ }^{32)}$ In addition to the effects of GSNO treatment, the inverse relationship between lysyl oxidase gene expression and cell proliferation is consistent with the findings of this and previous reports.

Endothelial dysfunction attenuates NO release, resulting in vascular diseases such as atherosclerosis. ${ }^{17)}$ It is possible that degeneration of elastic fiber formation is due to declining NO release. Mice that lack elastin gene undergo subendothelial cell proliferation and reorganization of smooth muscle. ${ }^{8)}$ Thus, elastic fiber formation was shown to regulate the proliferation of SMC in the development of atherosclerosis. In this present study, it suggests that increase of tropoelastin and LO mRNA expression by NO may cause increase of the elastin cross-linking processes in the aorta and may inhibit the developing arterial disease such as atherosclerosis.

Acknowledgments This work was supported in part by the Ministry of Education, Science, Sports, and Culture, Japan. We thank Kayo Tomioka, Saori Yoshioka, Rie Kobayashi, and Atsushi Ito for technical assistance.

\section{REFERENCES}

1) Rosenbloom J., Lab. Invest., 51, 605-623 (1984).

2) Liu J., Davidson J. M., Biochem. Biophys. Res. Commun., 154, 895901 (1988).

3) Wolfe B. L., Rich C. B., Goud H. D., Terpstra A. J., Bashir M., Rosenbloom J., Sonenshein G. E., Foster J. A., J. Biol. Chem., 268, 12418 12426 (1993).

4) Mauviel A., Chen Y. Q., Kahari V. M., Ledo I., Wu M., Rudnicka L., Uitto J., J. Biol. Chem., 268, 6520-6524 (1993).

5) Tokimitsu I., Tajima S., Nishikawa T., Biochem. Biophys. Res. Commun., 168, 850-856 (1990).

6) Hayashi A., Suzuki T., Wachi H., Tajima S., Nishikawa T., Murad S.,
Pinnell S. R., Arch. Biochem. Biophys., 315, 137-141 (1994).

7) Spencer A., Stahmann M. A., Atherosclerosis, 26, 139-150 (1977).

8) Li D. Y., Brooke B., Davis E. C., Mecham R. P., Sorensen L. K., Boak B. B., Eichwald E., Keating M. T., Nature (London), 393, 276-280 (1998).

9) Furchgott R. F., Zawadzki J. V., Nature (London), 288, 373-376 (1980).

10) Radomski M. W., Palmer R. M., Moncada S., Lancet, 2, 1057-1058 (1987).

11) Radomski M. W., Palmer R. M., Moncada S., Proc. Natl. Acad. Sci. U.S.A., 87, 5193-5197 (1990).

12) Kubes P., Suzuki M., Granger D. N., Proc. Natl. Acad. Sci. U.S.A., 88, 4651-4655 (1991).

13) Decatarina R., Libby P., Peng H.-B., Thannickai V. J., Rajavashisth T. B., Gimbrone M. A., Shin W. S., Liao J. K., J. Clin. Invest., 96, 60-68 (1995).

14) Pollman M. J., Yamada T., Horiuchi M., Gibbons G. H., Circ. Res., 79, 748 - 756 (1996).

15) Garg U. C., Hassid A., J. Clin. Invest., 83, 1774-1777 (1989).

16) Dubey R. K., Jackson E. K., Luscher T. F., J. Clin. Invest., 96, 141 149 (1995).

17) Yokoyama M., Hirata K., Miyake R., Akita H., Ishikawa Y., Fukuzaki H., Biochem. Biophys. Res. Commun., 168, 301-308 (1990).

18) Inoue N., Hirata K., Yamada M., Hamamori Y., Matsuda Y., Akita H., Yokoyama M., Circ. Res., 71, 1410-1421 (1992).

19) Miwa Y., Hirata K., Kawashima S., Akita H., Yokoyama M., Arterioscler. Thromb. Vasc. Biol., 17, 1561-1567 (1997).

20) Jansen A., Drazen J., Osborne J. A., Brown R., Loscalzo J., Stamler J. S., J. Pharmacol. Exp. Ther., 261, 154 (1992).

21) Oakes B. W., Batty A. C., Eur. J. Cell. Biol., 27, 34-46 (1982).

22) Tokimitsu I., Tajima S., Nishikawa T., Tajima M., Fukasawa T., Arch. Biochem. Biophys., 256, 455-461 (1987).

23) Cleveland D. W., Lopata M. A., MacDonald R. J., Cowan N. J., Rutter W. J., Kirschener M. W., Cell, 20, 95-105 (1980).

24) Wu Y., Rich C. B., Lincecum J., Trackman P. C., Kagan H. M., Foster J. A., J. Biol. Chem., 267, 24199-24206 (1992).

25) Tokimitsu I., Tajima S., J. Biochem., 115, 536-539 (1994).

26) Hayashi H., Wachi H., Tajima S., Biochim. Biophys. Acta, 1244, 325330 (1995).

27) Bressan G. M., Argos P., Stanley K. K., Biochemistry, 26, 1497-1503 (1987).

28) Baule V. J., Foster J. A., Biochem. Biophys. Res. Commun., 154, 1054-1060 (1988).

29) Kariya K., Kawahara Y., Araki S., Fukuzaki H., Takai Y., Atherosclerosis, 80, 143-147 (1989).

30) Mecham R. P., Levy B. D., Morris S. L., Madaras J. G., Wrenn D. S., J. Biol. Chem., 260, 3255-3258 (1985).

31) Ravid K., Smith-Mungo L. I., Zhao Z., Thomas K. M., Kagan H. M., J. Cell. Biochem., 75, 177-185 (1999).

32) Gacheru S. N., Thomas K. M., Murray S. A., Csiszar K., SmithMungo L., Kagan H. M., J. Cell. Biochem., 64, 1-13 (1997). 